\title{
Gold nanoparticles obtained by ns-pulsed laser ablation in liquids (ns-PLAL) are arranged in the form of fractal clusters
}

\author{
Luigi Gentile $\mathbb{C}$ - Helena Mateos $(\mathbb{D} \cdot$ - Antonia Mallardi $(\mathbb{D} \cdot$ - Marcella Dell'Aglio $(\mathbb{D} \cdot$ - Alessandro De \\ Giacomo (iD) - Nicola Cioffi (iD) Gerardo Palazzo (i)
}

Received: 4 June 2020 / Accepted: 4 January 2021 / Published online: 16 January 2021

(C) The Author(s) 2021

\begin{abstract}
Gold nanoparticles (AuNPs), synthesized by ns-pulsed laser ablation in liquid (ns-PLAL) in the absence of any capping agents, are potential model systems to study the interactions with biological structures unencumbered by interference from the presence of stabilizers and capping agents. However, several aspects of the physics behind these AuNPs solutions deserve a detailed investigation. The structure in solution of nsPLAL-synthesized AuNPs was investigated in solution by means of small-angle X-ray scattering (SAXS) and dynamic light scattering (DLS). Furthermore, the (dried) NPs have been examined using TEM. The analysis of the SAXS curve shows the presence of a large number of small aggregates with a fractal structure stabilized by strong long-range repulsive interactions. Fitting of the SAXS curve to a suitable "fractal model" allows the estimation of the features of the fractal including the fractal dimension $d=1.9$. The latter allows to estimate the fraction of light scattered by fractals of different
\end{abstract}

\footnotetext{
L. Gentile $(\bowtie) \cdot H$. Mateos · A. De Giacomo • N. Cioffi •

G. Palazzo

Department of Chemistry, University of Bari, and CSGI (Center for Colloid and Surface Science), via Orabona 4, 70125 Bari, Italy e-mail: luigi.gentile@uniba.it
}

\section{A. Mallardi}

c/o Chemistry Department, CNR-IPCF, Istituto per i Processi Chimico Fisici, Via Orabona 4, 70125 Bari, Italy

M. Dell'Aglio · A. De Giacomo • G. Palazzo $(\varangle)$ c/o Chemistry Department, CNR-NANOTEC, Istituto di Nanotecnologia, Via Orabona 4, 70125 Bari, Italy e-mail: gerardo.palazzo@uniba.it sizes and thus permits a fair comparison between the DLS and TEM data. Here, a stable abundant population of fractal clusters is reported reflecting a mechanism where primary AuNPs (size $7.6 \mathrm{~nm}$ ) are forced to aggregate forming clusters during the collapse of the cavitation bubble. When these clusters are released in the aqueous phase, their large negative charge builds up repulsive interactions that prevent cluster-cluster aggregation imparting colloidal stability.

Keywords Gold nanoparticles · Laser ablation in liquids · Small-angle X-ray scattering · Dynamic light scattering $\cdot$ Colloids $\cdot$ Cluster aggregation

\section{Introduction}

Nanoparticles (NPs) made of noble metals are wellknown key materials in many basic and applied research fields, spanning from catalysis, optics, fuel cell and medicine, just to cite a few (Speder et al. 2013, 2014; Silva et al. 2014; Ramalingam et al. 2014; Gellé and Moores 2019; Ferreira and Loh 2019; Ou et al. 2019).

Classical strategies to obtain metallic nanoparticles are based on the chemical reduction of corresponding oxidized cations by means of suitable reactants fulfilling the twofold roles of reductant and stabilizers. The presence of a stabilizing layer on the newborn particle is fundamental to prevent its endless growth (in such a case, the reactant acts as a capping agent) but also to avoid the coagulation of several NPs and the subsequent precipitation (Hamad-Schifferly 2014). However, the 
presence of stabilizing molecules on the surface of the particles is, unavoidably, a source of ambiguity in the mechanistic interpretation of the processes involving interactions between the NP and other molecules or surfaces. Very often, the reactivity of a given type of metallic NP depends strongly on the nature of the ligand used as a stabilizer. An interesting example is the interactions between gold nanoparticles (AuNPs) and phospholipid vesicles, mimetic of biological membranes. The interest in the study of such interactions is obviously related to their potential application in clinical translation of nanomedicine or at least in diagnostics (Maiolo et al. 2015; Paolini et al. 2016; Mallardi et al. 2018). It is known that the adsorption of AuNP to the vesicle surface and the subsequent effects that such a binding induces on the bilayer depend on the AuNP capping agents (Van Lehn et al. 2013; Wang and Liu 2015; Sugikawa et al. 2016; Montis et al. 2018; Liu et al. 2018; Kanwa et al. 2019; Pfeiffer et al. 2019). Browsing this literature, it becomes difficult to disentangle the contribution of the interactions between the membrane and the metallic core of NP from the aspects involving the capping agents.

In this respect, studies involving ligand-free nanoparticles without stabilizing capping molecules could be very useful.

This kind of NPs can be synthesized, without chemical reductants and/or capping agents, by means of the techniques based on laser ablation. As an example, pulsed laser deposition has been employed with success in the production of NP film (Dikovska et al. 2013; Smyth et al. 2013). This technique is based on the laser ablation of a target in vacuum condition in order to collect the NPs formed during the plasma expansion on a substrate suitably placed in the ablation chamber. Another attracting technique based on laser ablation is the "pulsed laser ablation in liquid" (PLAL) also known as "laser ablation synthesis in solution" (LASiS). PLAL is based on the laser pulsed irradiation of a bulk metal target submerged in a liquid (Dell'Aglio et al. 2015), and it allows the production of the colloidal solution with satisfactory properties in terms of size distribution and stability.

Upon interaction of the laser beam with the target surface, a laser-induced plasma is formed followed by a cavitation bubble formation. In a previous study, the plasma phase during ns-PLAL has been investigated by high temporally and spatially resolved optical emission spectroscopy (Dell'Aglio et al. 2019). It has been observed that as a consequence of the high density of the ablated material $\left(1020 \mathrm{~cm}^{-3}\right)$, in the colder zones of the plasma (around $4000 \mathrm{~K}$ ), NPs can grow until the equilibrium between NP evaporation and condensation is reached.

Other particles, with variable shape and size, can be formed at the border of the plasma, during the exchange of energy between the plasma and the surrounding water because of the fast cooling of the plasma material. Based on typical values of laser-induced plasma parameters during ablation in water (temperature between 4000 and $8000 \mathrm{~K}$ and electron number density around $1018 \mathrm{~cm}^{-3}$ ), electron charging of seeds and NPs occurs at picoseconds timescale in the plasma phase (Vogel et al. 1996).

When the internal energy of the plasma is exchanged with the surrounding water, a thin layer of water at high temperature and pressure is produced, and it expands inducing the cavitation bubble (Dell'Aglio and De Giacomo 2020). In this frame, charged NPs are delivered in the cavitation bubble with an excess of negative charge. During the bubble temporal evolution, if the electrostatic pressure of the cloud of NPs is higher than the pressure of the interface at the boundary of the cavitation bubble, NPs are released in solution; otherwise, they will be trapped inside the cavitation. These latter NPs, during the collapse stage, can experience a cavitation pressure much higher than the one originated by their Coulomb repulsion, and so they can rearrange in different shapes or can implant in the target (Dell'Aglio and De Giacomo 2020). At the end of the PLAL processes, different typologies of NPs can be found in the solution: (i) individual NPs grown in the plasma phase $(\sim 10 \mathrm{~nm})$; (ii) bigger particles (hundreds of $\mathrm{nm}$ ) formed at the border of the plasma; and (iii) clusters of NPs due to the interaction of the first kind of NPs during their releasing in solution (Dell'Aglio et al. 2015).

PLAL performed in water is a technique that allows the synthesis of metallic NP free from any capping layer. The interest for PLAL as a potential high-throughput synthetic method of metal NP generation has stimulated lively fundamental studies on the key ablation steps (the interactions among the laser-induced plasma, the solid metal and the liquid environment). At variance, several aspects of physics of the resulting NPs solution are often overlooked in the literature. For example, the colloidal stability of NP prepared by ns-PLAL in water is usually 
attributed to the presence of negative charges onto the surface of "naked" particles (Barcikowski and Compagnini 2013) (NPs have a strongly negative zeta potential), but the origin of those negative charges has only recently been addressed and is yet matter of debate (Jean-Philippe Sylvestre et al. 2004; Muto et al. 2007; Merk et al. 2014; Palazzo et al. 2017). In this contribution, we discuss the results of a small-angle X-ray scattering study performed on AuNPs solutions synthesized by ns-PLAL and the pieces of evidence of the arrangement of NPs in form of fractal clusters in the pristine solution.

This is the first time that the presence of stable clusters, of particles synthesized by ns-PLAL, is inferred on the basis of measurements performed in solution (SAXS and DLS). Furthermore, the presence of fractal structure is a feature that was not reported before for AuNP synthesized by laser ablation in water (there is only a report dealing with the outcome of ablation in supercritical trifluoromethane (Saitow et al. 2012)), although the production of aggregates has been clearly observed in several experiments (Ibrahimkutty et al. 2012; Reich et al. 2017; Dell'Aglio et al. 2019).

Fractal structures have been obtained on various substrates, by deposition of the target material during ablation in air (Celardo et al. 2017), in supercritical fluids (Saitow et al. 2012) and even in water (Musaev et al. 2009). However, pieces of evidence that this sort of structures exist also in the pristine ablation solution are limited to a report on the structure of silver nanoparticles synthesized in water by fs-PLAL, and also in such a case, the emphasis was on the structure deposited on solid surfaces (Santillán et al. 2015).

\section{Results and discussion}

The size distribution of NP prepared by PLAL is known to depend on the ion concentration of the ablation solution (Merk et al. 2014). Ablation in deionized water results in broad size distribution; while for too high ionic strength $(1 \mathrm{mM})$, the NPs coagulate immediately after their formation. Stable solutions of metal NPs are routinely synthesized by laser ablation in dilute aqueous solutions of salts at concentration about $0.1 \mathrm{mM}$. In order to have a fair comparison with our previous work, we have used as ablation solution $\mathrm{KCl} 10^{-4} \mathrm{M}$.

The resulting solution is characterized by the usual burgundy colour. A representative visible spectrum is shown in the inset of Fig. 1. Using the absorbance values at the wavelength of the surface plasmon $(520 \mathrm{~nm})$, at $450 \mathrm{~nm}$ and the calibration described elsewhere (Dell'Aglio et al. 2016), it is possible to estimate an average AuNP diameter of $17 \mathrm{~nm}$ and the NP concentration.

The colloidal stability of the AuNPs is electrostatic in nature; laser Doppler electrophoresis furnishes a broad distribution of zeta potential centred around $-64 \mathrm{mV}$ and with a width of $11 \mathrm{mV}$.

A time-saving sizing of the NPs can be obtained by dynamic light scattering (DLS). A representative autocorrelation function (ACF) is shown in Fig. 1.

The (field) ACF $g_{1}(q, \tau)$ is the customary output of DLS measurements, and it can be demonstrated (Berti and Palazzo 2014) that for a particle's system of equal diffusion coefficient (monodisperse in size), the ACF decays exponentially according to:

$g_{1}(q, \tau)=e^{-q^{2} D \tau}$

where $D$ is the mutual (or collective) diffusion coefficient reflecting the relaxation rate of the concentration fluctuations. For polydisperse samples, the scattered field ACF is no longer a single exponential, but rather it is the intensity-weighted sum of exponential decays,

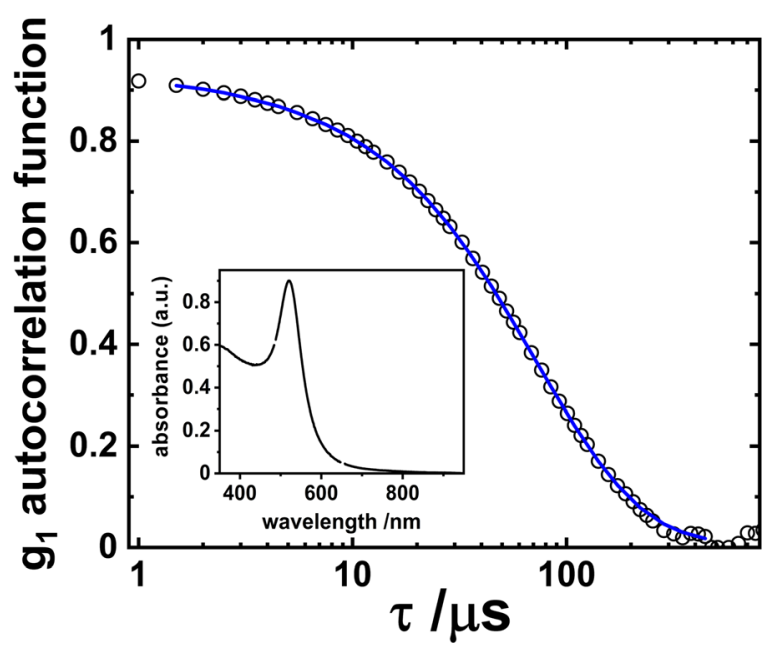

Fig. 1 Representative (field) autocorrelation function (ACF) collected in a DLS experiment on AuNPs synthesized by means of LAL. Points represent the experimental data; blue curve represents the ACF corresponding to the diffusion coefficient distribution $W(D)$ of Eq. (2). $W(D)$ was evaluated by numerical inversion of the Laplace transform in Eq. (2) by means of an NNLS routine. Inset: the corresponding absorbance spectrum. Conditions: $[\mathrm{AuNP}]=$ $1.5 \mathrm{nM}$ 
each accounting for the mutual diffusion of a population of particles:

$g_{1}(\tau)=\int_{0}^{\infty} W(D) e^{-q^{2} D \tau} d\left(q^{2} D\right)$

A robust way to account for this is according to the so-called cumulant expansion where the logarithm of the ACF is described by the first terms of the series:

$\ln \left[g_{1}(\tau)\right]=-\langle D\rangle_{Z} q^{2} \tau+\frac{\sigma^{2}}{2}\left(q^{2} \tau\right)^{2}+\ldots$

where $\langle D\rangle_{Z}$ is the average and $\sigma^{2}$ is the standard deviation of the diffusion-coefficient distribution W(D) entering Eq. (2). It should be noted that the average underlying $\langle D\rangle_{Z}$ is the so-called $z$-average, which emphasizes the largest particles from which an inverse, $z$-average particle diameter is calculated (Johnson and Gabriel 1994). In the case of the data shown in Fig. 1, the $z$-average diameter is $25 \mathrm{~nm}$, a value that could be consistent with the estimates from the absorption spectrum, taking into account that the $z$-average is biased towards the large particles.

The analysis of the DLS ACF in terms of truncated cumulant expansion (Eq. (3)) is a standard technique of general application, but the significance of the associated $z$-average size depends on the details of the size distribution function. For mono-modal, relatively narrow size distributions, the $z$-average is a meaningful descriptor while, in the case of coexistence of particles with an overall polymodal size distribution, it may not be so.

This can be an important issue in the case of particles synthesized by PLAL because the probability of secondary ablation is non-negligible, and this can lead to the fragmentation of primary particles in smaller ones.

Indeed, bimodal size distributions where small nanoparticles (few $\mathrm{nm}$ ) with narrow size distribution coexist with larger (more than $10 \mathrm{~nm}$ ) ones are often reported in the case of NPs produced by PLAL (Ibrahimkutty et al. 2012) (this depends also on the laser power and on the ablation time (Sportelli et al. 2018)).

To have insight on the details of the size distribution, few microlitres of the solution have been dried on TEM grids and observed using a transmission electron microscope.

Representative TEM micrographs are shown in Fig. $2 a$ and $b$ revealing the presence of spheroidal particles with diameters of the order of 10-20 nm. The histograms describing the size distribution are shown in the inset of Fig. 2a.

The largest majority of the nanoparticles $(80 \%)$ has a size below $17 \mathrm{~nm}$. It is important to highlight that, on the TEM grids, several particles are present in aggregates such as the one shown in Fig. 2a. In such cases, the size entering the statistics is that of the individual globules that are the constituent of the aggregate.

Since the sample preparation for conventional TEM requires the evaporation of the solvent, it is not possible to argue if the observed aggregates are a drying-induced artefact or they were present in the pristine dilute AuNP solution. Also, the evidence that in most cases the particles within an aggregate are "fused" is not significant since the AuNP coalescence in the solid state is an already reported phenomenon (Ingham et al. 2011).

To go into the details of the AuNPs structure in solution, we have performed SAXS experiments on liquid samples.

The SAXS intensity profile, $I(q)$ vs $q$, is shown in a linear-log representation in Fig. 3 from which some preliminary information can be extracted. First, the $I(q)$ is strongly affected by repulsive inter-particle interactions at low $q$ values (Guinier region); i.e. the structure factor, $S(q)$, cannot be neglected. This is consistent with the strong negative zeta potential $(-64 \mathrm{mV})$ measured.

Secondly, size polydispersity effects at high $q$ values have to be taken into account. For comparison in the inset of Fig. 3; the curve expected for non-interacting monodisperse spheres is also shown.

However, when the SAXS curve is examined in more detail with the help of a log-log representation like the one shown in Fig. 4a, it is clear that the experimental data can be hardly described in terms of simple spherical particles (albeit polydisperse). Indeed, while in the region at high $q$ values $\left(q>0.1 \AA^{-1}\right)$, where the oscillations are present, the overall decay of the $I(q)$ is $\propto q^{-4}$, the $I(q)$ at intermediate $q\left(0.01 \AA^{-1}<q<0.1 \AA^{-1}\right)$ still obeys a power law but with an exponent which is roughly halved (see the slopes of the power laws in Fig. 4b). This is an indication that some of the spherical particles are arranged in small aggregates with an inner fractal structure and a number $(N)$ of elementary particles of radius $R_{\mathrm{p}}$ given by

$N=\left(\frac{R_{g}}{R_{p}}\right)^{d}$ 

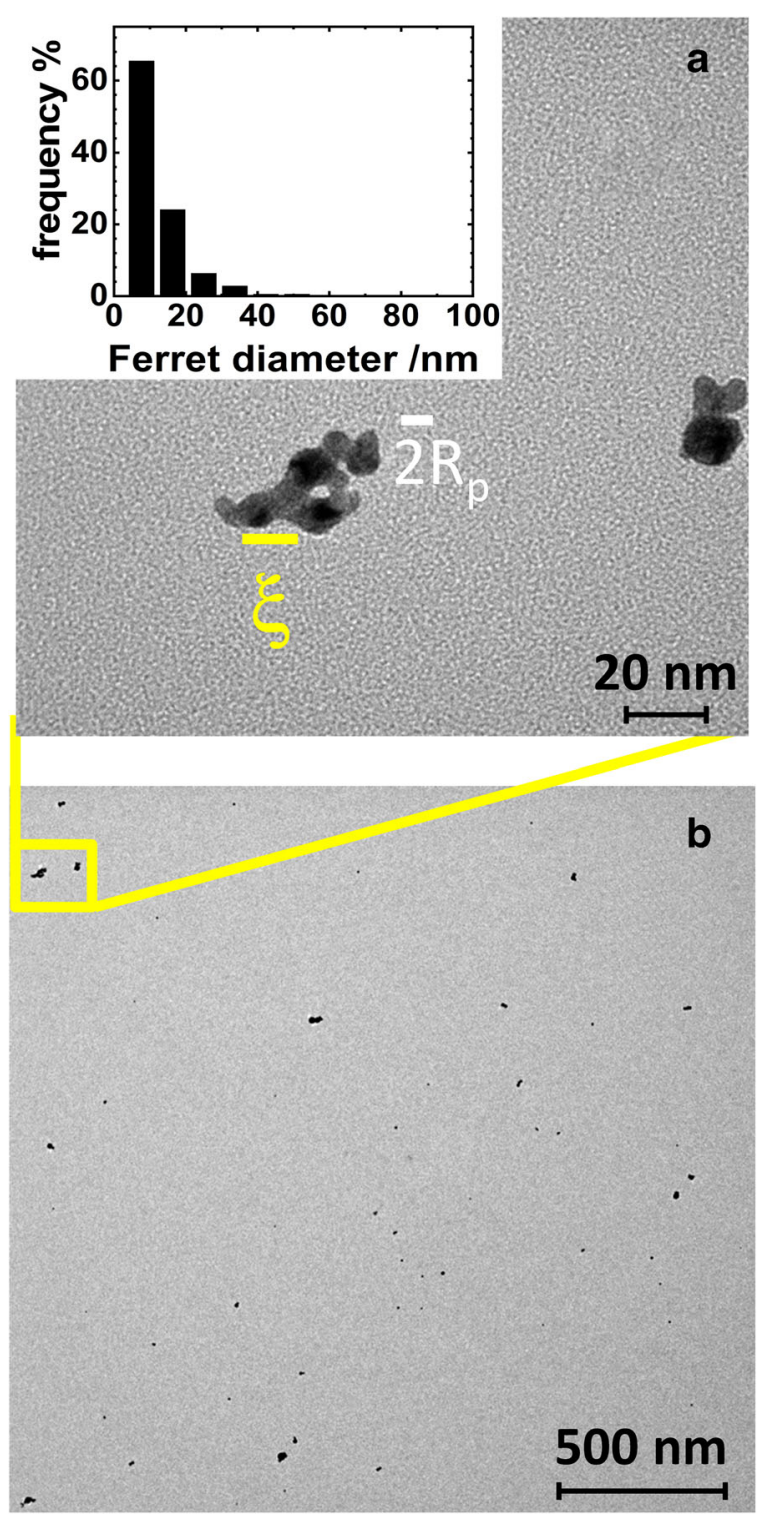

Fig. 2 a, b Representative TEM micrographs at different magnifications of AuNPs synthesized by PLAL. a is a zoom in the rectangular area (in yellow) of $\mathbf{b}$. Also shown in $\mathbf{a}$ are the diameter $\left(2 R_{\mathrm{p}}\right.$ in white) and the characteristic length ( $\xi$ in yellow), obtained from the fitting of the SAXS data; see text for details. The inset of a represents the size distribution of AuNPs

where $R_{g}$ is the gyration radius of the aggregate and $d$ represents the (mass) fractal dimension and is a measure of how efficiently the particles are packed.

Small-angle scattering methods are widely used to study the structure of aggregates of colloidal particles in solutions since they provide a direct measure of the fractal dimension because in the presence of fractal

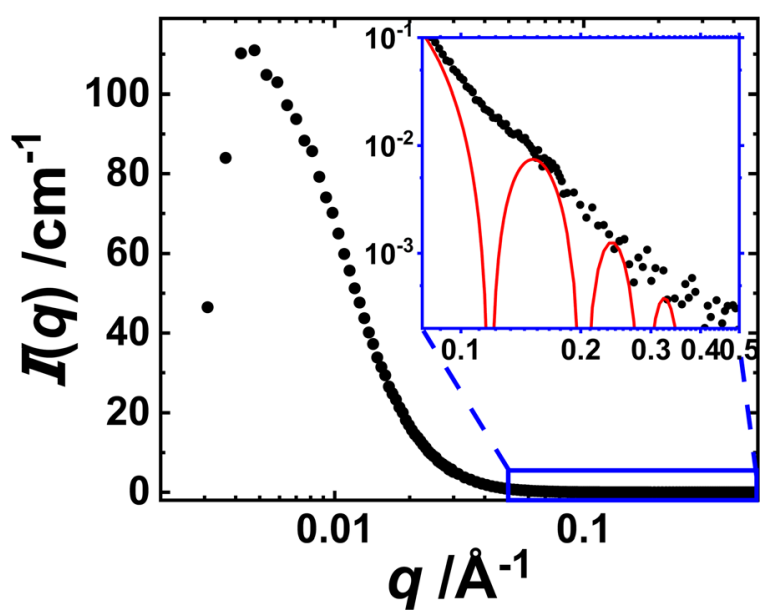

Fig. 3 SAXS curve for a solution of $[\mathrm{AuNP}]=16 \mathrm{nM}$. Inset: zoom of the region at $q>0.05 \AA^{-1}$; for the purpose of comparison, also the prediction for monodisperse sphere with radius $3.8 \mathrm{~nm}$ is shown as a red curve. The background subtraction was carefully made for the low q-region, where the resolution is lower. The $I(q)$ decrease at low $q$ is $\sim 50 \mathrm{~cm}^{-1}$ and cannot be explained by improper subtraction of the background having intensity $<16 \mathrm{~cm}^{-1}$

structures, the intensity of scattered radiation follows a power-law behaviour over some range of magnitude of the scattering vector, i.e. $I(q) \propto q^{-d}$ (Reidy et al. n.d.; Bushell et al. 2002; Wu et al. 2005; Ingham et al. 2011; Gentile et al. 2018).

The $q$ dependence of the intensity of the scattered radiation interference pattern can be always expressed through the relation:

$I(q)=N_{p} V_{p}^{2}\left(\rho_{p}-\rho_{\text {solvent }}\right)^{2} \cdot S(q) P(q)+B$

where $B$ is the background and $\rho_{\mathrm{s}}$ and $\rho_{\mathrm{p}}$ are the scattering length density of the solvent and of the homogeneous particle, respectively. In Eq. (5), the number density $(N p)$ of individual scatters (particles of volume $\left.V_{\mathrm{p}}\right)$ is $N_{\mathrm{p}}=\phi / V_{\mathrm{p}}$ where $\phi$ is the corresponding volume fraction. With respect to the following discussion about $\mathrm{X}$-ray and light scattering from fractal clusters, it is important to emphasize that $V_{\mathrm{p}}$ represents the portion volume of particles with scattering length $\rho_{\mathrm{p}}$, excluding the portion occupied by solvent (for example, in the case of a vesicle, $V_{\mathrm{p}}$ is the volume of the shell), and it is, thus, proportional to the particle mass $V_{\mathrm{p}} \propto M_{\mathrm{p}}$.

In Eq. (5), $P(q)$ is the form factor accounting for the intra-particle interference, while the structure factor $S(q)$ 

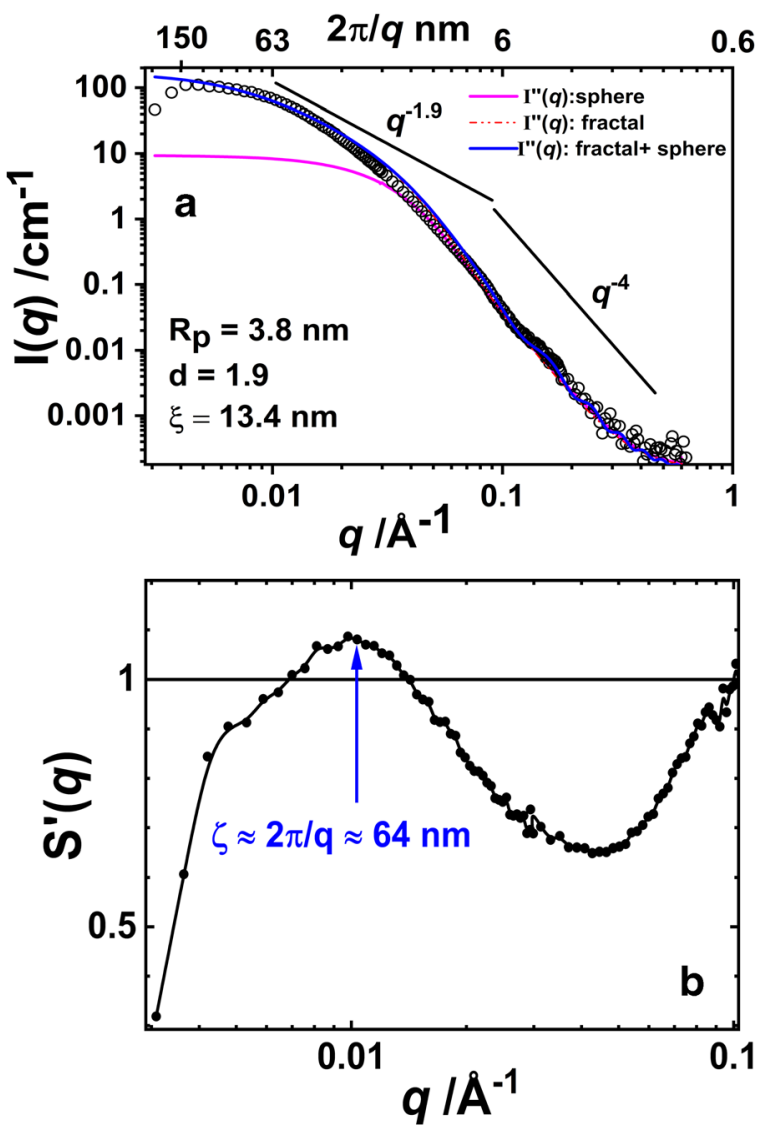

Fig. 4 a SAXS curve for a solution of $[\mathrm{AuNP}]=16 \mathrm{nM}$ in the log$\log$ representation (same data of Fig. 3). Empty dots are experimental data, the pink curve is the fit assuming a polydisperse sphere model, the dashed blue curve is the fit assuming the "fractal model" (Eqs. (6) and (7)), and the continuous red curve is the fit according to Eqs. (7) and (8) (i.e. fractal model + polydisperse sphere). The two straight lines denote the slope (in this representation) corresponding to Porod's law $\left(q^{-4}\right)$ and to a fractal dimension of $1.9\left(q^{-1.9}\right)$. (For further details, see text.) b Inter-aggregate/ particle structure factor $S^{\prime}(q)=(I(q)-B) / I^{\prime \prime}(q)$, where $I^{\prime \prime}(q)$ represents the fit according to Eqs. (7) and (8) (i.e. fractal model + polydisperse sphere)

represents the interference of waves scattered by different particles and is related to the existence of short-range ordering between particles. In the case of fractal aggregates, $S(q)$ can be factorized in the product of two contributions. The first, $S^{\prime}(q)$, is due to the customary interactions between aggregates ruled by the balance between repulsive and attractive interactions. The second, $S^{\prime \prime}(q)$, reflects the arrangement of the primary particles within a fractal aggregate.

Considering only this last term, and assuming that the fractal clusters are composed of spherical "building block" particles with radius $R_{p}$, Eq. (5) can be rewritten in terms of the "fractal model" (Mildner and Hall 1986) as follows:

$I^{\prime} \prime(q)=\phi V_{p}\left(\rho_{p}-\rho_{s}\right)^{2} \cdot S^{\prime} \prime(q) P(q)$

where $P(q)$ is the form factor of a sphere with radius $R_{p}$, while the structure factor describing the ordering of particles within a fractal, $S^{\prime \prime}(q)$, is given by the relation proposed by Teixeira (Teixeira 1988):

$S^{\prime} \prime(q)=1+\frac{d \Gamma(d-1) \sin \left[(d-1) \tan ^{-1}(q \xi)\right]}{\left(q R_{p}\right)^{d}\left(1+1 /(q \xi)^{2}\right)^{(d-1) / 2}}$

where $\Gamma(d-1)$ is the gamma function and $\xi$ is the correlation length representing the typical size of holes in the aggregate. A similar approach was adopted to interpret data coming from Ag nanoparticles prepared by fs-laser ablation (Santillán et al. 2015).

Equations (6) and (7) reasonably account for the experimental data (see the blue dashed line in Fig. 4) for $q>0.01 \AA^{-1}$. The agreement at high $q$ can be further improved by adding an additional spherical form factor to represent the single AuNPs nanoparticles (not involved in the aggregates) observed in the TEM micrographs along with the fractal aggregates. Thus obtaining:

$I^{\prime} \prime(q)=V_{p}\left(\rho_{p}-\rho_{s}\right)^{2} \cdot\left[\phi S^{\prime \prime}(q) P(q)+\phi_{p} P(q)\right]$

where $\phi_{p}$ is the volume fraction of these non-aggregates AuNPs and $P(q)$ is the form factor of spheres of radius $R_{p}$.

Equations (7) and (8) can be fitted to data collected at $q>0.005 \AA^{-1}$; a Schulz distribution with a polydispersity of 0.4 has been also applied to the sphere form factor (see the red curve in Fig. 5) to account for the spread in size. The resulting best-fit parameters are $\xi=$ $13.4 \mathrm{~nm}, d=1.9$, and $R_{\mathrm{p}}=3.8 \mathrm{~nm}$; the volume fraction of the NPs in the fractals was $\phi=6 \times 10^{-6}$, while the one of the individual NPs is $\phi_{\mathrm{p}}=1 \times 10^{-6}$. The order of magnitude of the overall volume fraction of $\operatorname{AuNP}(\phi+$ $\left.\phi_{\mathrm{p}}=7 \times 10^{-6}\right)$ is in agreement with the concentration of AuNPs estimated from the plasmonic absorption of gold nanoparticles (volume fraction $\sim 2 \times 10^{-5}$ ). The values of $R_{\mathrm{p}}$ and of a cluster correlation length $\xi$ that corresponds to 2-4 NP are consistent with TEM micrographs (bars representing the correlation length $\xi$ and the individual particle diameter $2 R_{\mathrm{p}}=7.6 \mathrm{~nm}$ are shown in Fig. $2 b$ for comparison). 
Strictly, Eqs. (7) and (8) refer to a system of particles where only the spherical shape of particles and their average distribution within the aggregates are taken into account. For this reason, we have marked by a double mark (") the intensity of the scattered radiation $\left(I^{\prime \prime}(q)\right)$ and the inter-particle structure factor within an aggregate $\left(S^{\prime \prime}(q)\right)$ to discriminate these quantities from the experimental quantities that take into account also the interactions among the aggregates $\left(S^{\prime}(q)\right)$. What is missing are the interactions between clusters and/or the single AuNP and the presence of a maximum in the experimental $I(q)$ indicates that such interactions exist, are repulsive, and are very strong.

In the absence of exhaustive modelling of the interactions among aggregates and/or single nanoparticles, the corresponding structure factor $S^{\prime}(q)$ can be determined by dividing the experimental data by the prediction of Eqs. (7) and (8). In Fig. 4b, we show such an inter-aggregate/particle structure factor $S^{\prime}(q)=(I(q)-$ $B) / I^{\prime \prime}(q)$; it is evident that the strongly repulsive interactions generate a structure factor peak that corresponds to a correlation distance, $\zeta=64 \mathrm{~nm}$, between aggregates and gold nanoparticles.

Having an estimate of the fractal dimension, we can try to compare the SAXS data with the DLS data.

According to Eq. (2), the ACF measured in a DLS experiment, $g 1(\mathrm{q})$, is the Laplace transform of the $W(D)$, the distribution function describing the amount of light scattered by particles with diffusion coefficient $D$. Such

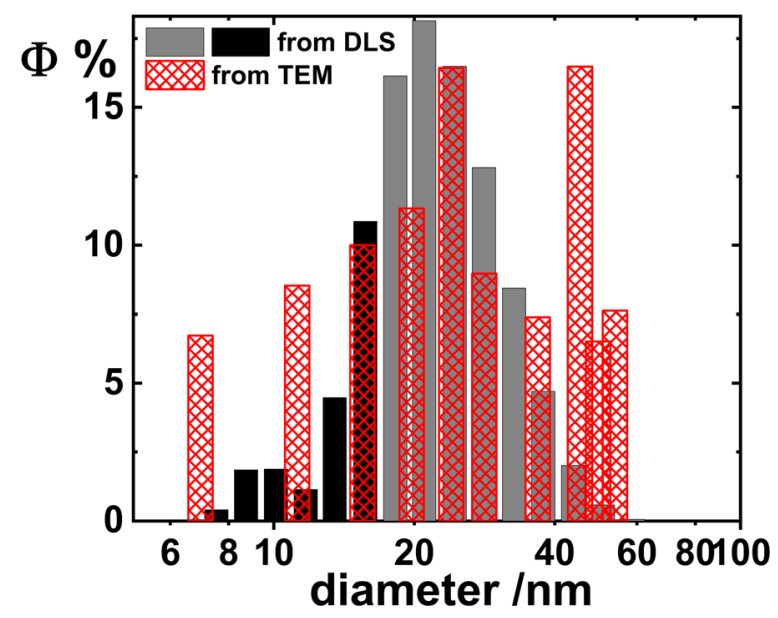

Fig. 5 Volume-weighted size distribution evaluated from the DLS data according to Eq. (11) and from TEM. Black histograms refer to free single nanoparticles, while the grey bars refer to the clusters. The red criss-crossed bars are from the analysis of the TEM micrographs; see text for details a $W(D)$ is easily mapped from the domain of diffusion coefficient to the domain of sizes applying the StokesEinstein equation and obtaining the so-called "intensity-weighted size distribution" $W\left(R_{\mathrm{h}}\right)$. Actually, $W\left(R_{h}\right)$ can be retrieved by inverting the Laplace transform in Eq. (2) applying a non-negative least-squares (NNLS) algorithm implemented in the software supplied by the manufacturer. $W\left(R_{\mathrm{h}}\right)$ represents the fraction of radiation (light) scattered by particles with a hydrodynamic size between $R_{\mathrm{h}}$ and $R_{\mathrm{h}}+d R_{\mathrm{h}}$ and depends on the number density of such particles and their size.

The dependence of $W\left(R_{\mathrm{h}}\right)$ on these parameters is given by Eq. (5), rewritten under the conditions that are relevant for a DLS experiment with visible radiation $(633 \mathrm{~nm})$ in which $q$ is constant and, since $q R_{\mathrm{g}} \sim 0$, both $P(0)=1$ and $S(0)=1$, this is:

$W\left(R_{\mathrm{h}}\right)=N_{\mathrm{c}} V_{\mathrm{c}}^{2}\left(\rho_{\mathrm{p}}-\rho_{\mathrm{s}}\right)^{2}$

In the above equation, the subscript $c$ emphasizes the circumstance that here we are dealing with clusters of elementary particles. The dependence on the cluster size $R_{\mathrm{h}}$ is included in the $V_{c}$ (the volume of the gold in the aggregate) that, for the reasons already discussed, is proportional to the cluster mass $V_{c} \propto M_{c}$ which, in turn, is proportional to the number of elementary particles in the cluster given by Eq. (4).

Strictly the hydrodynamic radius $R_{\mathrm{h}}$ measured by DLS is different from the $R_{\mathrm{g}}$, entering Eq. (4). However, it has been demonstrated that, for aggregates small enough, the dependence of scattered light intensity on aggregate size can be accounted for assuming $R_{\mathrm{h}} \sim R_{\mathrm{g}}$ (Pusey and Rarity 1987), and such an approach was successfully applied to the determination of fractal dimension (Pusey and Rarity 1987; Palazzo et al. 2010; Hanus et al. 2001).

Under the approximation $R_{\mathrm{h}} \sim R_{\mathrm{g}}$ it is possible to rewrite Eq. (9) as:

$W\left(R_{\mathrm{h}}\right)=N_{\mathrm{c}}\left(\frac{R_{\mathrm{h}}}{R_{\mathrm{p}}}\right)^{2 \mathrm{~d}}\left(\rho_{\mathrm{p}}-\rho_{\mathrm{s}}\right)^{2} \propto \phi\left(R_{\mathrm{h}}\right) R_{\mathrm{h}}{ }^{d}$

In the last right-hand term, we have exploited the relation $\phi \propto N_{\mathrm{c}} \cdot N$ that associates the cluster volume fraction to the number density and the number of elementary particles within that cluster. 
It is, therefore, possible to evaluate a volumeweighted size distribution as:

$\Phi\left(R_{\mathrm{h}}\right)=A \frac{W\left(R_{\mathrm{h}}\right)}{R_{\mathrm{h}}{ }^{d}}$

where $A$ is a normalization constant and $\Phi\left(R_{\mathrm{h}}\right)$ is correlated to the volume fractions $\phi$ and $\phi_{\mathrm{p}}$ entering the Eq. (8) used to analyse the SAXS data by the following equations:

$$
\int_{\text {clusters }} \Phi d R_{\mathrm{h}}=\frac{\phi}{\phi+\phi_{\mathrm{p}}} \text { and } \int_{\mathrm{NPs}} \Phi d R_{\mathrm{h}}=\frac{\phi_{\mathrm{p}}}{\phi+\phi_{\mathrm{p}}}
$$

The volume-weighted size distribution evaluated according to Eq. (11), using a fractal dimension of $d=1.9$ obtained from the SAXS analysis, is shown in Fig. 5.

According to the TEM sizing (Fig. 3), the vast majority of NPs has diameters $\leq 16 \mathrm{~nm}$; therefore, we have divided the histograms in Fig. 5 into two categories: those with $2 R_{\mathrm{h}}<16 \mathrm{~nm}$ belong to single NPs (black bars), and those with $2 R_{\mathrm{h}}>16 \mathrm{~nm}$ belong to clusters (grey bars). It is thus possible to perform the integrations in Eq. (12) with the outcome that the fraction of nonaggregate NPs probed by DLS is $11 \%$ of the total. This is a value very close to the result obtained through the analysis of SAXS curve for which $\phi_{p}$ accounts for $14 \%$ of the total particles.

Equation (4) foretells that the gold volume in an aggregate made of $\mathrm{N}$ elementary particles scales as $R_{\mathrm{g}}{ }^{d}$, and this allows the estimation of $\Phi$ also on the basis of electron microscopy results.

Accordingly, the TEM micrographs have been reanalysed, but this time, when an aggregate formed by several particles is present, its overall size has been determined in terms of (maximum) Feret's diameter (the longest distance between any two points in the aggregate), and assuming the Feret's diameter as a reasonable estimate of the aggregate gyration size, the volumeweighted size distribution, shown in Fig. 5 as red criss-crossed histograms, is obtained. The agreement between DLS and TEM is, once the fractal dimension is properly taken into account, considerable.

It should be stressed that despite a fractal dimension of 1.9 is often reported for fractals growing through DLCA, the small clusters here observed are stable for weeks from the preparation. This can be hardly reconciled with the orthodox DLCA models that lead to an irreversible formation of infinite aggregates.
How is it possible to resolve such an aggregation with the strong repulsive interactions testified by the peak in the inter-particle structure factor of Fig. $4 b$ ? Or, in other words, why the attractive inter-particle forces that drive the cluster formation do not lead to irreversible aggregation of all the particles and clusters?

Currently, the origin of the negative zeta potential found in noble metal NPs, prepared by PLAL, is still a matter of debate. Two (nonexclusive) mechanisms could be of interest here: the presence of excess electrons formed within the plasma phase (the large size of NPs considerably reduces the Born's energy of the extra charges) (Palazzo et al. 2017; Mateos et al. 2020a) and the absorption of anions from the aqueous solution (Merk et al. 2014).

Most likely, the initial fractal aggregates are formed during the collapse stage of the bubble. As already reported in Ref. (Dell'Aglio et al. 2019), the laserinduced plasma during the initial steps of the plasma evolution produces NPs that are strongly charged by adsorption of the free electrons abundant in the plasma atmosphere. Then, during the bubble collapse phase, the produced NPs experience the backpressure of the cavitation environment (Dell'Aglio et al. 2019) that compresses the NPs clouds and can induce the agglomeration of the NPs in clusters.

One could speculate that, once in the aqueous solution at atmospheric pressure, the clusters formed in the ablation steps are stabilized against further aggregation by the repulsive interactions due to their negative charge (does not matter if ascribed to extra electrons or to adsorbed anions) confirmed by the large negative zeta potential experimentally observed.

It is interesting to note that this mechanism fits the results reported in Ibrahimkutty et al. (2012) and Reich et al. (2017), where with in situ SAXS experiment performed during the NS-PLAL processes, the creation of "primary" smaller NPs is subsequently followed by the formation of the aggregates after the maximum expansion of the cavitation bubble.

\section{Conclusions}

In this contribution, we have investigated the structure of AuNPs solution synthesized by laser ablation by a multi-techniques approach. Analysis of SAXS and DLS results self-consistently indicates that, in the AuNPs solution synthesized by laser ablation, the majority of 
the nanoparticles are present in the form of small clusters (with a fractal dimension $d=1.9$ ) made of few small particles $\left(R_{\mathrm{p}}=3.2 \mathrm{~nm}\right)$ characterized by a correlation length $\xi \sim 13 \mathrm{~nm}$ that accounts for $\sim 85 \%$ of the gold mass (of course the number of clusters is much lower). Once the fractal dimension is taken into account, it is possible to retrieve the volume (or weight) size distribution from DLS data and to compare it with the analogous distribution obtained from the analysis of TEM micrographs. The data coming from the two different techniques are in good agreement, thus validating the overall scenario.

In hindsight, almost all the published TEM micrographs of ablated AuNPs show visible clusters, sometimes large, sometimes small (2-4 particles), but this has been always interpreted as a consequence of the drying process (Jean-Philippe Sylvestre et al. 2004; Muto et al. 2007; Merk et al. 2014). The results of the present study suggest that further aggregation due to the sample processing on the carbon-coated grid is negligible.

Even though the average size of the aggregates is small, some consideration on their fractal dimension should be highlighted. The fractal dimension of $d=1.9$ is slightly higher with respect to tabulated values for the 3D diffusion-limited cluster aggregation (DLCA) and lower with respect to the $3 \mathrm{D}$ reaction-limited cluster aggregation (RLCA) (Odriozola et al. 2001) The value 1.9 might indicate a DLCA regime where particles can rearrange in the forming cluster, probably in a short time. However, a real DLCA mechanism will lead to continuous aggregation of the clusters to form flocks and to a rapid flock sedimentation, an event undetected over the timescale of months.

The stability of clusters with a fractal dimension of 1.9 is puzzling because such a value is often reported for fractals growing indefinitely through DLCA (Wu et al. 2005). In the present case, instead, the clusters are stabilized by strong repulsive electrostatic interactions. To reconcile these two conflicting pieces of evidence, we hypothesize the clusters are formed during the ablation and precisely throughout the bubble collapse phase (Dell'Aglio et al. 2019). During such a step, the huge backpressure of the cavitation environment compresses the NPs ensemble and might induce the aggregation of the NPs in clusters. In this respect, further characterization of such metallic small clusters could give insight into the different stages of laser ablation in water.
On a wider perspective, the full characterization of the structure and interactions present in solution of these ligand-free AuNPs by NS-PLAL is preliminary to the subsequent investigation on the interactions between AuNPs and biological membranes without the interference of the capping agents.

\section{Experimental}

AuNP synthesis and preliminary characterization

To produce AuNPs by NS-PLAL, a ns NdYAG (Quanta System PILS-GIANT) laser was focused on Au target located on the bottom of a cuvette filled with $3 \mathrm{~mL}$ of Milli-Q water. The laser, with a nominal pulse duration of $8 \mathrm{~ns}$, operates with a $1064 \mathrm{~nm}$ laser wavelength, with a fluence of $64 \mathrm{~J} / \mathrm{cm}^{2}$ and a laser frequency of $10 \mathrm{~Hz}$. The laser beam was focused by using $5-\mathrm{cm}$ focal lens, and the focal plane of the laser beam was put inside the target, in order to get a laser crater on the target surface with a diameter of $1 \pm 0.2 \mathrm{~mm}$. The gold target was purchased from Kurt J. Lesker Company. AuNPs colloidal solutions were produced by adding to the Milli-Q water $\mathrm{KCl} 100 \mu \mathrm{M}$.

The production rate in this experiment determined by measuring the concentration of NPs in the solution with SPR (Dell'Aglio and De Giacomo 2020) is about $4 \times$ $10^{10}$ NPs per laser shot. This means that this rate of production already takes into account all the processes involved in the laser ablation in liquid, including secondary laser particle interaction such as melting and fragmentation (Lorazo et al. 2006). A further discussion on these processes would be out of scope in the present paper that is focused in the characterization of the particle already released in water but interested reader can refer to (Tsuji 2002).

The NP concentration increases with the ablation time until eventually reaching a steady value of $16 \mathrm{nM}$ because of the increasing concentration of AuNPs within the laser radiation path, thus producing an attenuation of the incident beam (Yang 2012).

The vis spectrum of AuNPs in the region between 350 and $900 \mathrm{~nm}$ was registered using an Agilent 8453 UV-visible spectrophotometer.

AuNPs solutions were characterized by DLS and by laser Doppler electrophoresis (LDE) measurements using a Zetasizer-Nano ZS from Malvern Instruments. Instrumental conditions are as in references (Mateos 
et al. 2020b) (DLS) and (Mateos et al. 2019) (LDE); the zeta potential was subsequently evaluated from the electrophoretic mobility measured by LDE according to the Hückel approximation.

The average NP size, evaluated over more than 50 syntheses, is reproducible within $20 \%$ when evaluated by DLS and by $10 \%$ when evaluated from the SPR.

\section{Electron microscopy}

Colloidal samples for TEM characterization were directly deposited onto carbon-coated $\mathrm{Cu}$ grids (300 mesh, Agar Scientific). All samples were observed at $120 \mathrm{kV}$, with an FEI Tecnai T12 TEM.

The size distribution of the NPs was obtained by measuring the diameter of at least $400 \mathrm{NPs}$ using Image J software. The pictures were processed to obtain binary images to which the threshold was adjusted to visualize the NPs. To differentiate the NPs from the agglomerates, the images were "watershed" segmented and the range of analysis particles was set up from 10 pixels per square to infinity.

The average NP size, evaluated over 10 syntheses by TEM, is reproducible within $15 \%$.

\section{SAXS}

SAXS measurements were performed using a SAXS Lab Ganesha 300XL instrument (SAXSLAB ApS, Skovlunde, Denmark), a pinhole collimated system equipped with a Genix 3D X-ray source (Xenocs SA, Sassenage, France). The scattering intensity $I(q)$ was recorded with the detector placed at two sample-todetector distances, yielding scattering vectors $(q)$ of 0.004-0.6 $\AA^{-1}$. Samples were sealed at room temperature in a $1.5-\mathrm{mm}$ diameter quartz capillary (Hilgenberg $\mathrm{GmbH}$, Malsfeld, Germany). In all cases, the temperature was controlled by an external recirculating water bath fixed to $25^{\circ} \mathrm{C}$, with an accuracy of approximately $0.2{ }^{\circ} \mathrm{C}$. The two-dimensional (2D) scattering pattern was recorded using a 2D $300 \mathrm{k}$ Pilatus detector (Dectris Ltd., Baden, Switzerland) and radially averaged using SAXSGui software to obtain $I(q)$.

For SAXS experiments, the highest concentration $([\mathrm{AuNP}]=16 \mathrm{nM})$ is used in order to improve the signal-to-noise ratio; lowest NP concentrations return the same fractal dimensions, but the curves are too noisy to have a reliable fit of the whole data to Eq. (8). The measured scattering curves were corrected for solvent scattering.

Acknowledgements Open Access funding provided by Università degli Studi di Bari Aldo Moro.

Funding This project has received funding from the European Union's ERASMUS+ "Staff Mobility for Training" program and from the POR PUGLIA FESR-FSE 2014/2020 Research for Innovation (REFIN)-Codice Pratica: 7BDC8679, and partial financial support from the Center for Colloid and Surface Science (CSGI).

\section{Compliance with ethical standards}

Conflict of interest The authors declare that they have no conflict of interest.

Open Access This article is licensed under a Creative Commons Attribution 4.0 International License, which permits use, sharing, adaptation, distribution and reproduction in any medium or format, as long as you give appropriate credit to the original author(s) and the source, provide a link to the Creative Commons licence, and indicate if changes were made. The images or other third party material in this article are included in the article's Creative Commons licence, unless indicated otherwise in a credit line to the material. If material is not included in the article's Creative Commons licence and your intended use is not permitted by statutory regulation or exceeds the permitted use, you will need to obtain permission directly from the copyright holder. To view a copy of this licence, visit http://creativecommons.org/licenses/by/4.0/.

\section{References}

Barcikowski S, Compagnini G (2013) Advanced nanoparticle generation and excitation by lasers in liquids. Phys Chem Chem Phys 15:3022-3026

Berti D, Palazzo G (2014) Scattering of radiation. In: Berti D, Palazzo G (eds) Colloidal foundations of Nanoscience. Elsevier, Amsterdam, p 177

Bushell GC, Yan YD, Woodfield D, Raper J, Amal R (2002) On techniques for the measurement of the mass fractal dimension of aggregates. Adv Colloid Interf Sci 95:1-50

Celardo GL, Archetti D, Ferrini G, Gavioli L, Pingue P, Cavaliere E (2017) Evidence of diffusive fractal aggregation of $\mathrm{TiO} 2$ nanoparticles by femtosecond laser ablation at ambient conditions - IOPscience. Mater Res Express 4:

Dell'Aglio M, De Giacomo A (2020) Plasma charging effect on the nanoparticles releasing from the cavitation bubble to the solution during nanosecond pulsed laser ablation in liquid. Appl Surf Sci 515:146031. https://doi.org/10.1016/j. apsusc.2020.146031

Dell'Aglio M, Gaudiuso R, De Pascale O, De Giacomo A (2015) Mechanisms and processes of pulsed laser ablation in liquids 
during nanoparticle production. Appl Surf Sci 348:4-9. https://doi.org/10.1016/j.apsusc.2015.01.082

Dell'Aglio M, Mangini V, Valenza G, de Pascale O, de Stradis A, Natile G, Arnesano F, de Giacomo A (2016) Silver and gold nanoparticles produced by pulsed laser ablation in liquid to investigate their interaction with ubiquitin. Appl Surf Sci 374:297-304. https://doi.org/10.1016/j.apsusc.2015.11.253

Dell'Aglio M, Motto-Ros V, Pelascini F, Gornushkin IB, de Giacomo A (2019) Investigation on the material in the plasma phase by high temporally and spectrally resolved emission imaging during pulsed laser ablation in liquid (PLAL) for NPs production and consequent considerations on NPs formation. Plasma Sources Sci Technol 28:085017. https://doi.org/10.1088/1361-6595/AB369B

Dikovska AO, Alexandrov MT, Atanasova GB, Tsankov NT, Stefanov PK (2013) Silver nanoparticles produced by PLD in vacuum: role of the laser wavelength used. Appl Phys A Mater Sci Process 113:83-88. https://doi.org/10.1007 /s00339-013-7834-9

Ferreira GA, Loh W (2019) Planet-satellite nanostructures based on block copolymer-surfactant nanoparticles surfacedecorated with gold and silver: a new strategy for interfacial catalysis adv mater interfaces. Planet-s:6. https://doi. org/10.1002/admi.201900348

Gellé A, Moores A (2019) Plasmonic nanoparticles: photocatalysts with a bright future. Curr Opin Green Sustain Chem 15:60-66

Gentile L, Wang T, Tunlid A, Olsson U, Persson P (2018) Ferrihydrite nanoparticle aggregation induced by dissolved organic matter. J Phys Chem A 122:7730-7738. https://doi. org/10.1021/acs.jpca.8b05622

Hamad-Schifferly K (2014) Stability of dispersions and interactions. In: Berti D, Palazzo G (eds) Colloidal foundations of Nanoscience. Elsevier, Amsterdam, pp 33-46

Hanus LH, Hartzler RU, Wagner NJ (2001) Electrolyte-induced aggregation of acrylic latex. 1: dilute particle concentrations. Langmuir 17:3136-3147. https://doi.org/10.1021/la000927c

Ibrahimkutty S, Wagener P, Menzel A, Plech A, Barcikowski S (2012) Nanoparticle formation in a cavitation bubble after pulsed laser ablation in liquid studied with high time resolution small angle x-ray scattering. Appl Phys Lett 101:103104. https://doi.org/10.1063/1.4750250

Ingham B, Lim TH, Dotzler CJ, Henning A, Toney MF, Tilley RD (2011) How nanoparticles coalesce: an in situ study of au nanoparticle aggregation and grain growth. Chem Mater 23: 3312-3317. https://doi.org/10.1021/cm200354d

Johnson CS Jr, Gabriel D (1994) Laser light scattering. Dover Pubblications, New York

Kanwa N, Patnaik A, De SK et al (2019) Effect of surface ligand and temperature on lipid vesicle-gold nanoparticle interaction: a spectroscopic investigation. Langmuir 35:1008-1020. https://doi.org/10.1021/acs.langmuir.8b03673

Liu X, Li X, Xu W, Zhang X, Huang Z, Wang F, Liu J (2018) Subangstrom gold nanoparticle/liposome interfaces controlled by halides. Langmuir 34:6628-6635. https://doi.org/10.1021 /acs.langmuir.8b01138

Lorazo P, Lewis LJ, Meunier M (2006) Thermodynamic pathways to melting, ablation, and solidification in absorbing solids under pulsed laser irradiation. Phys Rev B - Condens Matter Mater Phys 73:1-22. https://doi.org/10.1103 /PhysRevB.73.134108
Maiolo D, Paolini L, Di Noto G et al (2015) Colorimetric nanoplasmonic assay to determine purity and titrate extracellular vesicles. Anal Chem 87:4168-4176. https://doi. org/10.1021/ac504861d

Mallardi A, Nuzziello N, Liguori M, Avolio C, Palazzo G (2018) Counting of peripheral extracellular vesicles in multiple sclerosis patients by an improved nanoplasmonic assay and dynamic light scattering. Colloids Surfaces B Biointerfaces 168:134-142. https://doi.org/10.1016/j.colsurfb.2018.02.006

Mateos H, Valentini A, Robles E, Brooker A, Cioffi N, Palazzo G (2019) Measurement of the zeta-potential of solid surfaces through laser Doppler electrophoresis of colloid tracer in a dip-cell: survey of the effect of ionic strength, $\mathrm{pH}$, tracer chemical nature and size. Colloids Surfaces A Physicochem Eng Asp 576:82-90. https://doi.org/10.1016/j. colsurfa.2019.05.006

Mateos H, Picca RA, Mallardi A, Dell'Aglio M, de Giacomo A, Cioffi N, Palazzo G (2020a) Effect of the surface chemical composition and of added metal cation concentration on the stability of metal nanoparticles synthesized by pulsed laser ablation in water. Appl Sci 10:1-12. https://doi.org/10.3390 /APP10124169

Mateos H, Valentini A, Colafemmina G, Murgia S, Robles E, Brooker A, Palazzo G (2020b) Binding isotherms of surfactants used in detergent formulations to bovine serum albumin. Colloids Surfaces A Physicochem Eng Asp:598. https://doi.org/10.1016/j.colsurfa.2020.124801

Merk V, Rehbock C, Becker F, Hagemann U, Nienhaus H, Barcikowski S (2014) In situ non-DLVO stabilization of surfactant-free, plasmonic gold nanoparticles: effect of Hofmeister's anions. Langmuir 30:4213-4222. https://oi. org/10.1021/la404556a

Mildner DFR, Hall PL (1986) Small-angle scattering from porous solids with fractal geometry

Montis C, Generini V, Boccalini G, Bergese P, Bani D, Berti D (2018) Model lipid bilayers mimic non-specific interactions of gold nanoparticles with macrophage plasma membranes. $\mathrm{J}$ Colloid Interface Sci 516:284-294. https://doi.org/10.1016/j. jcis.2018.01.064

Musaev OR, Midgley AE, Wrobel JM, Yan J, Kruger MB (2009) Fractal character of titania nanoparticles formed by laser ablation. J Appl Phys 106:054306. https://doi.org/10.1063 $/ 1.3208058$

Muto H, Yamada K, Miyajima K, Mafuné F (2007) Estimation of surface oxide on surfactant-free gold nanoparticles laserablated in water. J Phys Chem C 111:17221-17226. https://doi.org/10.1021/jp075582m

Odriozola G, Tirado-Miranda M, Schmitt A, Martínez López F, Callejas-Fernández J, Martínez-García R, Hidalgo-Álvarez R (2001) A light scattering study of the transition region between diffusion- and reaction-limited cluster aggregation. J Colloid Interface Sci 240:90-96. https://doi.org/10.1006 /jcis.2001.7687

Ou J, Zhou Z, Chen Z, Tan H (2019) Optical diagnostic based on functionalized gold nanoparticles. Int J Mol Sci 20:4346. https://doi.org/10.3390/ijms20184346

Palazzo G, Lopez F, Mallardi A (2010) Effect of detergent concentration on the thermal stability of a membrane protein : the case study of bacterial reaction center solubilized by. Biochim Biophys Acta - Proteins Proteomics 1804:137146. https://doi.org/10.1016/j.bbapap.2009.09.021 
Palazzo G, Valenza G, Dell'Aglio M, De Giacomo A (2017) On the stability of gold nanoparticles synthesized by laser ablation in liquids. J Colloid Interface Sci 489:47-56. https://doi. org/10.1016/j.jcis.2016.09.017

Paolini L, Zendrini A, Di Noto G et al (2016) Residual matrix from different separation techniques impacts exosome biological activity. Sci Rep 6:1-11. https://doi.org/10.1038/srep23550

Pfeiffer T, De Nicola A, Montis C et al (2019) Nanoparticles at biomimetic interfaces: combined experimental and simulation study on charged gold nanoparticles/lipid bilayer interfaces. J Phys Chem Lett 10:129-137. https://doi.org/10.1021 /acs.jpclett.8b03399

Pusey PN, Rarity JG (1987) Measurement of the hydrodynamic fractal dimension of aggregating polystyrene spheres. Mol Phys 62:411-418. https://doi.org/10.1080 /00268978700102281

Ramalingam V, Rajaram R, Premkumar C et al (2014) Biosynthesis of silver nanoparticles from deep sea bacterium Pseudomonas aeruginosa JQ989348 for antimicrobial, antibiofilm, and cytotoxic activity. J Basic Microbiol 54: 928-936. https://doi.org/10.1002/jobm.201300514

Reich S, Schönfeld P, Wagener P, Letzel A, Ibrahimkutty S, Gökce B, Barcikowski S, Menzel A, dos Santos Rolo T, Plech A (2017) Pulsed laser ablation in liquids: impact of the bubble dynamics on particle formation. J Colloid Interface Sci 489:106-113. https://doi.org/10.1016/j. jcis.2016.08.030

Reidy RF, Allen AJ, Krueger S (n.d.) Small angle neutron scattering characterization of colloidal and fractal aerogels

Saitow KI, Okamoto Y, Yano YF (2012) Fractal of gold nanoparticles controlled by ambient dielectricity: synthesis by laser ablation as a function of permittivity. J Phys Chem C 116: 17252-17258. https://doi.org/10.1021/jp304109h

Santillán JMJ, Fernández van Raap MB, Mendoza Zélis P et al (2015) $\mathrm{Ag}$ nanoparticles formed by femtosecond pulse laser ablation in water: self-assembled fractal structures. J Nanoparticle Res:17. https://doi.org/10.1007/s11051-015-2894-8

Silva BR, Lunardi CN, Araki K, Biazzotto JC, da Silva RS, Bendhack LM (2014) Gold nanoparticle modifies nitric oxide release and vasodilation in rat aorta. J Chem Biol 7:5765. https://doi.org/10.1007/s12154-014-0109-x

Smyth CA, Mirza I, Lunney JG, McCabe EM (2013) Surfaceenhanced Raman spectroscopy (SERS) using Ag nanoparticle films produced by pulsed laser deposition. Appl Surf Sci 264:31-35. https://doi.org/10.1016/j.apsusc.2012.09.078

Speder J, Altmann L, Roefzaad M, Bäumer M, Kirkensgaard JJK, Mortensen K, Arenz M (2013) Pt based PEMFC catalysts prepared from colloidal particle suspensions-a toolbox for model studies. Phys Chem Chem Phys 15:3602-3608. https://doi.org/10.1039/c3cp50195g
Speder J, Altmann L, Bäumer M, Kirkensgaard JJK, Mortensen K, Arenz M (2014) The particle proximity effect: from model to high surface area fuel cell catalysts. RSC Adv 4:1497114978. https://doi.org/10.1039/c4ra00261j

Sportelli MC, Clemente M, Izzi M, Volpe A, Ancona A, Picca RA, Palazzo G, Cioffi N (2018) Exceptionally stable silver nanoparticles synthesized by laser ablation in alcoholic organic solvent. Colloids Surfaces A Physicochem Eng Asp 559:148-158. https://doi.org/10.1016/j.colsurfa.2018.09.046

Sugikawa K, Kadota T, Yasuhara K, Ikeda A (2016) Anisotropic self-assembly of citrate-coated gold nanoparticles on fluidic liposomes. Angew Chemie - Int Ed 55:4059-4063. https://doi.org/10.1002/anie.201511785

Jean-Philippe Sylvestre, Suzie Poulin, Andrei V. Kabashin, et al (2004) Surface chemistry of gold nanoparticles produced by laser ablation in aqueous media. https://doi.org/10.1021 /JP047134+

Teixeira J (1988) Small-angle scattering by fractal systems. J Appl Crystallogr 21:781-785. https://doi.org/10.1107 /S0021889888000263

Tsuji T (2002) Preparation of silver nanoparticles by laser ablation in solution. Appl Surf Sci 202:80-85

Van Lehn RC, Atukorale PU, Carney RP et al (2013) Effect of particle diameter and surface composition on the spontaneous fusion of monolayer-protected gold nanoparticles with lipid bilayers. Nano Lett 13:4060-4067. https://doi.org/10.1021 /n1401365n

Vogel A, Busch S, Parlitz U (1996) Shock wave emission and cavitation bubble generation by picosecond and nanosecond optical breakdown in water. J Acoust Soc Am 100:148-165. https://doi.org/10.1121/1.415878

Wang F, Liu J (2015) Self-healable and reversible liposome leakage by citrate-capped gold nanoparticles: probing the initial adsorption/desorption induced lipid phase transition. Nanoscale 7:15599-15604. https://doi.org/10.1039/c5 nr04805b

Wu H, Xie J, Lattuada M, Morbidelli M (2005) Scattering structure factor of colloidal gels characterized by static light scattering, small-angle light scattering, and small-angle neutron scattering measurements. Langmuir 21:3291-3295. https://doi.org/10.1021/la047403n

Yang G (2012) Laser ablation in liquids: principles and applications in the preparation of nanomaterials. Taylor \& Francis, Stanford

Publisher's note Springer Nature remains neutral with regard to jurisdictional claims in published maps and institutional affiliations. 